\title{
DOES THE POSTOPERATIVE ANALGESIA AFFECT MORBIDITY IN BARIATRIC SURGERY? A SINGLE-CENTRE RETROSPECTIVE REVIEW.
}

\section{Background and goal of study}

Uneventful postoperative recovery in morbidly obese patients undergoing bariatric surgery may offer challenges to anesthesiologists. This study aimed to investigate a possible relationship between different acute-pain analgesic therapies and postoperative morbidity and mortality in bariatric surgery.

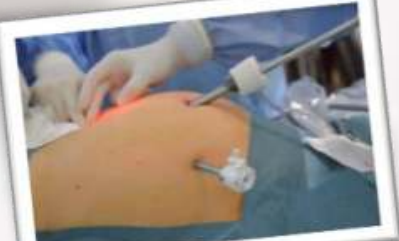

\section{Material and methods}

This was a retrospective observational study of patients $(n=60)$ who underwent laparoscopic sleeve gastrectomy. The subjects were classified into 4 groups according to different analgesic therapies for acute postoperative pain: GROUP A -intravenous morphine infussion (1.5 mg/h; $\mathrm{n}=18)$; GROUP B - intravenous tramadol (100 mg every 8 hours; $n=14$ ); GROUP C - intravenous NSAIDs (dexketoprofen 50 mg every 8 hours; $n=13$ ) or GROUP D - epidural fentanyl/levobupivacaine infusion (fentanyl $2 \mathrm{mcg} / \mathrm{ml}$; levobupivacaine $0.125 \% ; 8 \mathrm{ml} / \mathrm{h} ; \mathrm{n}=15$ ).

The following parameters were recorded: demographic characteristics, Body Mass Index (BMI), ASA physical status classification system, postoperative complications, URPA stay, ICU stay and total hospital stay.

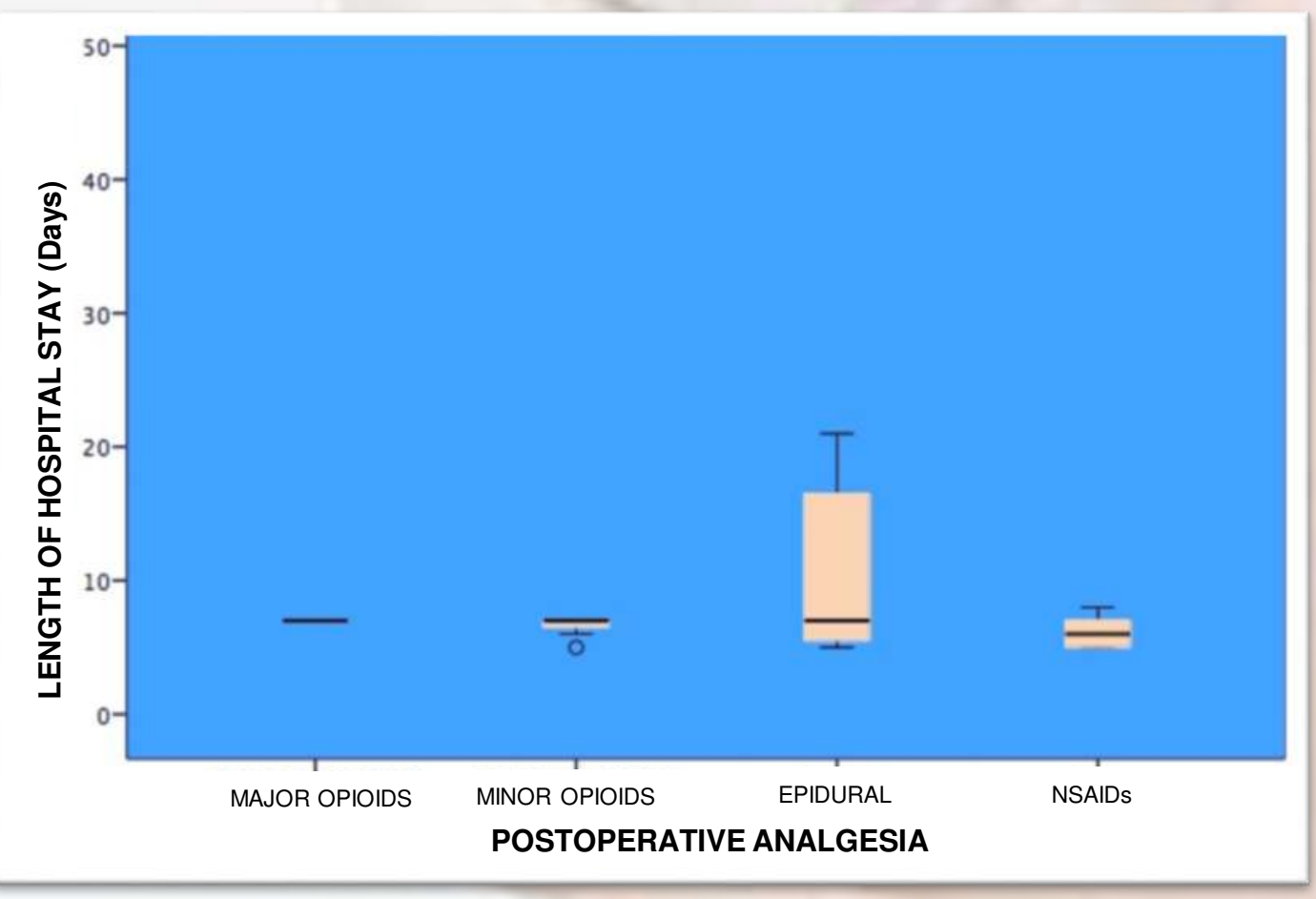

\section{Results and discussion}

Morbid obesity prevalence in the sample was higher in women $(65 \%)$. However, groups can be considered demographically homogeneous. With regard to hospital length of stay (URPA stay, ICU stay and total hospital stay) there were no statistically significant differences among analgesic therapies $(\mathrm{p} 1=0.161 ; \mathrm{p} 2$ $=0.194 ; \mathrm{p} 3=0.367$ ). The incidence of postoperative nausea or vomiting was higher in intravenous-opioid therapies $(11 \%$ tramadol; 16\% morphine).

\begin{tabular}{|c|c|c|}
\hline Complications & $\mathbf{n}$ & Description \\
\hline $\begin{array}{c}\text { Infectious } \\
\text { complications }\end{array}$ & 2 & $\begin{array}{l}\text { Intra-abdominal abscess and surgical wound } \\
\text { infection }\end{array}$ \\
\hline $\begin{array}{l}\text { Respiratory } \\
\text { complications }\end{array}$ & 2 & Acute hypercapnic respiratory failure (x2) \\
\hline $\begin{array}{l}\text { Haemodynamic } \\
\text { complications }\end{array}$ & 3 & $\begin{array}{l}\text { Haemodynamic instability and vasoactive drugs } \\
\qquad(x 2)\end{array}$ \\
\hline $\begin{array}{c}\text { Surgical } \\
\text { complications }\end{array}$ & 4 & $\begin{array}{c}\text { Septic shock folowing anastomotic leak (x 2) } \\
\text { Hemoperitoneum (x2) }\end{array}$ \\
\hline $\begin{array}{c}\text { Other } \\
\text { complications }\end{array}$ & 2 & Postoperative nausea and vomiting (PONV). \\
\hline Mortality & 1 & \\
\hline
\end{tabular}

\section{Conclusion}

None of these therapies has shown to increase morbidity, mortality or length of hospital stay in the sample. Therefore, it is a multiple-choice question with several correct answers. Nevertheless, we need a larger sample size to gain power and obtain new conclusions. The integration of multimodal analgesia techniques with a multidisciplinary rehabilitation program in bariatric surgery may enhance recovery, reduce hospital stay, and facilitate early convalescence.

\section{REFERENCES:}

1. Vieito M, Hernández J, Santiveri X, García Ch, Maestre P, Villalonga A y cols.: Morbimortalidad anestésica-quirúrgica en 60 pacientes intervenidos de cirugía bariátrica. Rev Esp Anestesiol Reanim. 2002; 49:365-372.

2. Silva PT, Patias LD, Alvarez Gda C, Kirsten VR, Colpo E, Moraes CM. Profile of patients who seek the bariatric surgery. Arra Bras Cir Dig. $2015 ; 28: 270-3$. 